\title{
Intra-abdominal extravasation complicating parenteral nutrition in infants
}

\author{
S Nour, J W L Puntis, M D Stringer
}

\begin{abstract}
Two infants receiving total parenteral nutrition via a central venous catheter positioned in the inferior vena cava developed an acute abdomen secondary to extravasation of the infusate. The presence of an associated abdominal mass necessitated a laparotomy in one patient. Both infants recovered completely after the catheter had been removed. (Arch Dis Child 1995; 72: F207-F208)
\end{abstract}

Keywords: parenteral nutrition, central venous catheter.

Over the past two decades parenteral nutrition has become an essential part of the management of infants with a wide variety of medical and surgical conditions. Its limitations and complications are well recognised but intraabdominal extravasation of parenteral nutrition fluid has rarely been documented. We describe two patients who developed an acute abdomen as a result of this complication.

\section{Case reports}

A 30 week gestational age boy weighing $1 \mathrm{~kg}$ and requiring ventilatory support after developing respiratory distress syndrome was parenterally fed from day 3. The tip of a percutaneous fine bore Silastic catheter (Epicutaneo-Cava-Catheter), introduced without difficulty via the right long saphenous vein at the knee, was sited in the inferior vena cava (figure). Free bidirectional flow through the line was confirmed and intravenous feeding using a standard nutritional regimen was started with normal line pressures.

On day 3 the infant began a seven day course of treatment with vancomycin for possible coagulase negative staphylococcal sepsis (positive peripheral blood cultures). After a pulmonary haemorrhage he required increased ventilatory support and then developed abdominal distension and bile-stained gastric aspirates. By day 17 he had a tender indurated mass in the right iliac fossa and increasing abdominal distension. Serial abdominal radiographs revealed no intramural gas but fixed loops of dilated bowel were noted in the right iliac fossa. A provisional diagnosis of necrotising enterocolitis was made but he continued to deteriorate despite treatment with broad spectrum antibiotics, and a laparotomy was performed.

At surgery, about $100 \mathrm{ml}$ of sterile chylous peritoneal fluid, with an identical appearance to parenteral nutrition solution, was found in association with an oedematous retroperitoneal mass in the right iliac fossa. There were no other abnormalities. The ascites was aspirated, the abdomen closed with drainage, and the silastic catheter removed. The patient made a rapid recovery with resolution of all abdominal signs. The catheter tip and ascitic fluid were sterile and the inferior vena cava was patent on a subsequent ultrasound scan. Retrospective review of line infusion pressures failed to reveal abnormally high pressures.

A 6 month old boy with the VATER complex required temporary parenteral nutrition while undergoing diagnosis and treatment of severe tracheomalacia and gastro-oesophageal reflux. Intravenous feeding was delivered via a $5 \mathrm{Fr}$ polyurethane catheter (Cook, Denmark) introduced percutaneously into the right femoral vein, using a Seldinger technique and with the catheter tip positioned in the inferior vena cava. Two days later, he developed abdominal distension and acute respiratory distress. An abdominal radiograph showed a relatively gasless abdomen and an abdominal ultrasound scan confirmed a large amount of peritoneal fluid. Chylous ascites was drained by paracentesis, and the femoral catheter, which could be flushed but not aspirated, was removed without recurrent problems. The patient responded well to aortopexy and was thriving at the time of writing.

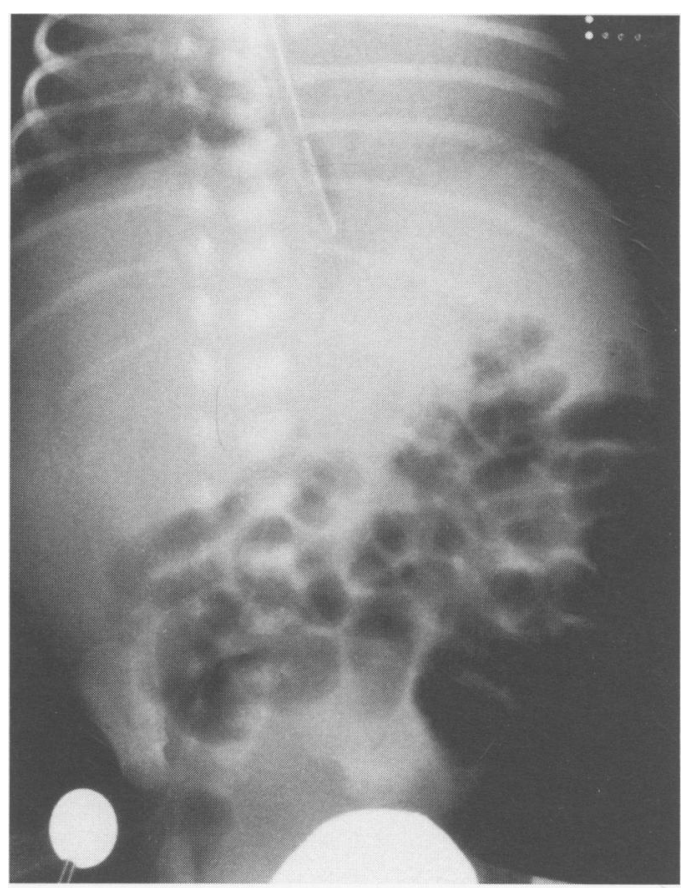

The percutaneous catheter is positioned in the inferior vena cava. 


\section{Discussion}

The documented complications of parenteral nutrition delivered via central venous catheters are legion but can be broadly classified as mechanical, thrombotic, infective and metabolic. Extravasation of parenteral nutrition fluid from such catheters, either from direct vascular perforation or in association with thrombosis, is a recognised cause of pleural effusion ${ }^{12}$ and pericardial tamponade. ${ }^{3}$ Intraabdominal extravasation has rarely been reported in infants. ${ }^{14-6}$ Our two patients presented acutely two days to two weeks after starting parenteral feeding via lower limb catheters, each composed of different materials. In both cases the catheter tip was positioned in the lower inferior vena cava.

Localised chemical or septic phlebitis and subsequent catheter erosion is the most likely cause of extravasation; previous reports have suggested that non-occlusive mural thrombo$\operatorname{sis}^{1}$ and, in very low birthweight infants, coagulase negative staphylococcal infection, ${ }^{6}$ are important precursors. Thrombotic occlusion of the inferior vena cava is not a prerequisite; it was not shown in our first patient and intra-abdominal parenteral nutrition extravasation was not observed in any of the 15 infants with proved inferior vena cava catheter thrombosis reported by Mulvihill and Fonkalsrud. ${ }^{7}$

Both of our patients developed abdominal distension and respiratory distress, but the presence of a palpable abdominal mass and of dilated fixed loops of bowel in the premature infant indicated the possibility of necrotising enterocolitis for which a laparotomy was undertaken. In the few published cases either localised retroperitoneal fluid collection or generalised ascites have been described in association with abdominal distension and abdominal wall erythema, leading to exploratory laparotomy in some cases. ${ }^{6}$ The use of abdominal ultrasound or an intravenous contrast study through the catheter may avoid the need for surgery if this potential complication of parenteral nutrition is suspected. ${ }^{156}$

Once diagnosed, intra-abdominal parenteral nutrition extravasation responds well to catheter removal ${ }^{156}$ together with paracentesis if there is respiratory compromise. The complication can be prevented by optimal placement of the catheter tip in the mid right atrium; if the catheter can be advanced only into the inferior vena cava, the risk may be reduced by positioning the tip above the renal veins. ${ }^{16}$

1 Spriggs DW, Brantley RE. Thoracic and abdominal extravasation: a complication of hyperalimentation in infants. $A m$ 7 Roentgenol 1977; 128: 419-22.

2 Seguin JH. Right sided hydrothorax and central venous catheters in extremely low birth weight infants. $A m \mathcal{F}$ Perinatol 1992; 9: 154-9.

3 Giacoia GP. Cardiac tamponade and hydrothorax as complications of central venous parenteral nutrition in infants.

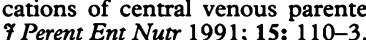

4 Axelsson CK, Knudsen FU. Catheter induced ascites - an unusual complication of parenteral feeding. Intensive Care Med 1978; 4: 91-2.

5 Krasna IH, Krause T. Life-threatening fluid extravasation of central venous catheters. $\mathcal{F}$ Pediatr Surg 1991; 26: 1346-8

6 Bansal V, Strauss A, Gyepes M, Kanchanapoom V. Central line perforation associated with Staphylococcus epidermidis infection. F Pediatr Surg 1993; 28: 894-7.

7 Mulvihill SJ, Fonkalsrud EW. Complications of superior versus inferior vena cava occlusion in infants receiving central total parenteral nutrition. $\mathcal{F}$ Pediatr Surg 1984; 19: 752-7. 